\title{
Moms in motion: weight loss intervention for postpartum mothers after gestational diabetes: a randomized controlled trial
}

Briana J. Stith', Samantha M. Buls', Sarah A. Keim ${ }^{1,2,3}$, Stephen F. Thung ${ }^{4}$, Mark A. Klebanoff ${ }^{2,3,4,5}$, Mark B. Landon ${ }^{4}$, Steven G. Gabbe ${ }^{4}$, Kajal K. Gandhi ${ }^{6}$ and Reena Oza-Frank ${ }^{4,7^{*}}$

\begin{abstract}
Background: Up to $50 \%$ of women with gestational diabetes mellitus (GDM) will receive a diagnosis of type 2 diabetes mellitus (T2DM) within a decade after pregnancy. While excess postpartum weight retention exacerbates T2DM risk, lifestyle changes and behavior modifications can promote healthy postpartum weight loss and contribute to T2DM prevention efforts. However, some women have difficulty prioritizing self-care during this life stage. Efficacious interventions that women can balance with motherhood to reduce T2DM risk remain a goal. The objective of the Moms in Motion study is to evaluate the efficacy of a simple, novel, activity-boosting intervention using ankle weights worn with daily activities during a 6-month postpartum intervention among women with GDM. We hypothesize that women randomized to the 6-month intensity-modifying intervention will (1) demonstrate greater weight loss and (2) greater improvement in body composition and biomarker profile versus controls.
\end{abstract}

Methods: This study will be a parallel two-arm randomized controlled trial $(n=160)$. Women will be allocated 1:1 to an ankle weight intervention group or a standard-of-care control group. The intervention uses ankle weights (1.1 $\mathrm{kg}$ ) worn on each ankle during routine daily activities (e.g., cleaning, childcare). Primary outcomes include pre- and post-assessments of weight from Visit 2 to Visit 3. Secondary outcomes include body composition, glycemia (2-h, $75 \mathrm{~g}$ oral glucose tolerance test), and fasting insulin. Exploratory outcomes include energy expenditure, diet, and psychosocial well-being.

Discussion: Beyond the expected significance of this study in its direct health impacts from weight loss, it will contribute to exploring (1) the mechanism(s) by which the intervention is successful (mediating effects of energy expenditure and diet on weight loss) and (2) the effects of the intervention on body composition and biomarkers associated with insulin resistance and metabolic health. Additionally, we expect the findings to be meaningful regarding the intervention's effectiveness on engaging women with GDM in the postpartum period to reduce T2DM risk.

\footnotetext{
* Correspondence: reena.oza-frank@odh.ohio.gov

${ }^{4}$ Department of Obstetrics and Gynecology, College of Medicine, The Ohio

State University, 370 W. 9th Avenue, Columbus, OH 43210, USA

${ }^{7}$ Ohio Department of Health, 246 N High Street, Columbus, OH 43215, USA

Full list of author information is available at the end of the article
}

(c) The Author(s). 2021 Open Access This article is licensed under a Creative Commons Attribution 4.0 International License, which permits use, sharing, adaptation, distribution and reproduction in any medium or format, as long as you give appropriate credit to the original author(s) and the source, provide a link to the Creative Commons licence, and indicate if changes were made. The images or other third party material in this article are included in the article's Creative Commons licence, unless indicated otherwise in a credit line to the material. If material is not included in the article's Creative Commons licence and your intended use is not permitted by statutory regulation or exceeds the permitted use, you will need to obtain permission directly from the copyright holder. To view a copy of this licence, visit http://creativecommons.org/licenses/by/4.0/. The Creative Commons Public Domain Dedication waiver (http://creativecommons.org/publicdomain/zero/1.0/) applies to the data made available in this article, unless otherwise stated in a credit line to the data. 
Trial registration: The ClinicalTrials.gov Identifier, is NCT03664089. The trial registration date is September 10, 2018. The trial sponsor is Dr. Sarah A. Keim.

Keywords: Gestational diabetes mellitus, Clinical trial, Exercise, Physical activity, Lifestyle intervention, Prevention of type 2 diabetes mellitus, Weight loss

\section{Background}

The prevalence of gestational diabetes mellitus (GDM) has increased steadily in recent decades, affecting at least $7 \%$ of pregnant women in the United States (US) [1]. Up to $50 \%$ of women with GDM will develop type 2 diabetes mellitus (T2DM) within a decade after delivery [2]. Postpartum weight loss can reduce the risk of developing T2DM [3]. For instance, one study reported that each 10-pound increment of weight retained after pregnancy is associated with a $27 \%$ higher risk of T2DM [2], and a $2.5 \%$ decrease from baseline body weight is associated with a $60 \%$ reduction in T2DM incidence [4]. However, postpartum women have difficulty prioritizing self-care during this life stage [5]. Less than 33\% of women with GDM undergo a postpartum oral glucose tolerance test, which is the first step in assessing postpartum T2DM risk and initiating prevention efforts [6].

Existing interventions for women with GDM require considerable time outside of already hectic schedules to change multiple behaviors. Arguably, the most effective weight loss interventions implement physical activity and dietary changes simultaneously. However, a sequential approach may be less overwhelming, place fewer demands on a person's ability to change their behavior, require less effort [7], and be better suited for developing stronger habits long term [8]. Streamlined interventions may be even more feasible for women of low socioeconomic status.

Three US-based studies have attempted to translate the Diabetes Prevention Program (DPP) for women with GDM [9-11] by modifying it to address lack of time as the strongest perceived barrier to behavior change [12, 13] (e.g., through telephone $[9,10]$, web [11], and mail [9]). In two of the studies, the intervention group retained less weight $[10,11]$, but this translated to only $\sim 25 \%$ of participants, reflecting low levels of participation and adherence to the interventions. Two of these studies resulted in slight diet improvements $[9,11]$, and one resulted in increased self-reported physical activity [14]. Even if these interventions had shown more promising results, they were time- and resource-intensive, limiting scalability.

A recent pilot randomized controlled trial (RCT) tested the efficacy of a digital health support program to improve postpartum behaviors and glucose tolerance testing among women with GDM [15]. Participants received an activity monitor and motivational text messages integrated with their activity data. Although postpartum testing increased and the text messages yielded positive feedback from the participants, there was no significant difference between the control and intervention groups in dietary or physical activity goals achieved, and only $45 \%$ of participants completed the program. Another study examined weight loss and physical activity using a 3-month, web-based behavioral intervention amongst overweight or obese women with GDM [16]. The intervention consisted of a pedometer program and nutrition counseling with a sample of 28 Caucasian women and 3 women of Asian descent. Despite the small sample size, engaging postpartum women in behavior modification was challenging. Like previous studies, common barriers including lack of time, no childcare, and difficulty implementing lasting lifestyle changes reportedly affected all stages of the study and contributed to their difficulty recruiting and high attrition rate.

The lack of desired results and inconsistent success with previous studies is likely due to incomplete attention to aligning the type of intervention and intervention intensity to barriers postpartum women have to joining and adhering to lifestyle behavior change. It is evident that efficacious interventions to reduce T2DM risk that women can balance with motherhood remain a goal. Our intervention attempts to address this deficiency by adding more intensity to routine daily activities. New mothers are already engaged in an unavoidable lifestyle modification - they spend much of their time engaged in light-intensity physical activity such as cooking, cleaning, and childcare [17-21]. These same activities alone lower blood glucose [22]. We hypothesize that by increasing the intensity of these usual, daily activities, our intervention aims to maximize and increase energy expenditure to efficiently promote weight loss, an essential step towards reducing T2DM risk.

\section{Methods/design \\ Specific aims}

1) Compare the efficacy of an intervention focused on intensity modification during daily activities versus standard recommendations on measured weight loss (primary outcome).

2) Evaluate the impact of the intervention on body composition (i.e., \% body fat, waist-hip ratio) and 
biomarkers associated with insulin resistance (i.e., glucose, insulin, HOMA, HbA1c, adiponectin, leptin) and metabolic health (i.e., lipids, blood pressure, hsCRP) (secondary outcomes).

3) Examine energy expenditure and diet as potential mediators to better understand the mechanisms behind greater weight loss in the intervention group (exploratory outcomes).

\section{Study design}

The Moms in Motion study will be a parallel two-arm RCT to evaluate the efficacy of a simple, novel, physical activity-boosting intervention on postpartum weight loss among women with GDM.

\section{Setting}

Women will be recruited from Ohio State University Wexner Medical Center Maternal-Fetal Medicine Diabetes in Pregnancy Clinics in Columbus, Ohio, USA. These clinics serve a large and diverse population, including both privately insured and publicly insured women referred from a large catchment area.

\section{Participants}

Participants will be 160 women diagnosed with GDM during their current pregnancy. GDM is diagnosed with an oral glucose tolerance test using the CarpenterCoustan criteria [23], typically between 24- and 28weeks gestation. Testing may occur sooner if a patient has a higher risk of developing GDM.

This sample size was selected based on preliminary data and previous experience from our pilot study. We determined that a sample size of 160 women would be adequate and obtainable due to the number of eligible women we estimate to recruit and enroll, also considering an assumed $20 \%$ attrition rate. The recruitment clinics saw 419 unique patients with GDM in 2014. Based on preliminary data and previous experience, we estimate $\geq 80 \%$ of those women will be eligible $(n=335)$, of which $>90 \%$ will enroll resulting in a potential of $n=$ $300 /$ year. Our hypothesis is that women in the intervention will lose more weight than women in the control group. Sample size calculations were based on the difference in the pattern of weight means at study baseline and study endpoint (6-month intervention). Our preliminary data from our pilot studies indicate the intervention group mean weight loss from baseline to month 6 was 6.3 pounds vs. 3.9 pounds in the control group. Using linear mixed effect modeling to detect differences in mean weight change, we estimate an effect size (Cohen's d) of 0.63. Using a more conservative $d=0.50$, we estimate $n=64 /$ group $(N=128)$ for $80 \%$ power. Assuming a $20 \%$ drop out rate, we need $n=80 /$ group ( $N=$
160), well within the estimated number of eligible women visiting the recruitment sites.

Eligible women will include those that provide written informed consent at 30-40 weeks' gestation or 7-14 days postpartum, 18 years of age or older, English-speaking, plan to remain in the local area for the duration of the study and are physically capable of engaging in moderate physical activity. Women who are not English-speaking will be excluded because the resources for translating and validating standardized questionnaires for use in other languages are not currently available. Women who have a prior type 1 or type 2 diabetes diagnosis, are surrogate gestational carriers, are pregnant with multiples, deliver prior to 35 weeks' gestation, have a prepregnancy BMI of less than 18.5 (underweight), or live more than 35 miles away from the Ohio State University Wexner Medical Center (Columbus, Ohio) will be ineligible.

Figure 1 provides the CONSORT flow diagram for the study, which is ongoing. It includes the total intended number for randomization. We do not have a total intended number for patients screened.

\section{Recruitment and informed consent}

Clinic staff will distribute informational flyers to participants deemed eligible based on the inclusion and exclusion criteria and who attend the Diabetes in Pregnancy education class (first clinical point of contact after a GDM diagnosis). Study staff will then use the electronic medical record (EMR) and clinic schedule to approach eligible participants during their prenatal appointment to discuss the study and administer informed consent. Women who express interest will be eligible to consent at 30-40 weeks' gestation prior to completing any studyrelated procedures. If they are not able to consent before delivery, they will have the opportunity during Visit 1, a home visit completed 7-14 days postpartum. Participants will receive a copy of Nationwide Children's Hospital's Notice of Privacy Practices at the point of consent, detailing their rights as a participant. All patients will have the potential to visit the clinic more than once after enrollment, allowing research staff to monitor subsequent visits of enrolled participants to remind them about the study and receive updates.

\section{Procedures: study visits and other contacts}

There will be three study visits. Table 1 details the measures included in each visit. Table 2 shows the timeline for which these visits will be completed and the acceptable windows for these visits to be completed.

\section{Visit 1 and run-in period}

The first study visit (Visit 1) will take place in the participant's home 7-14 days postpartum. Study staff will 


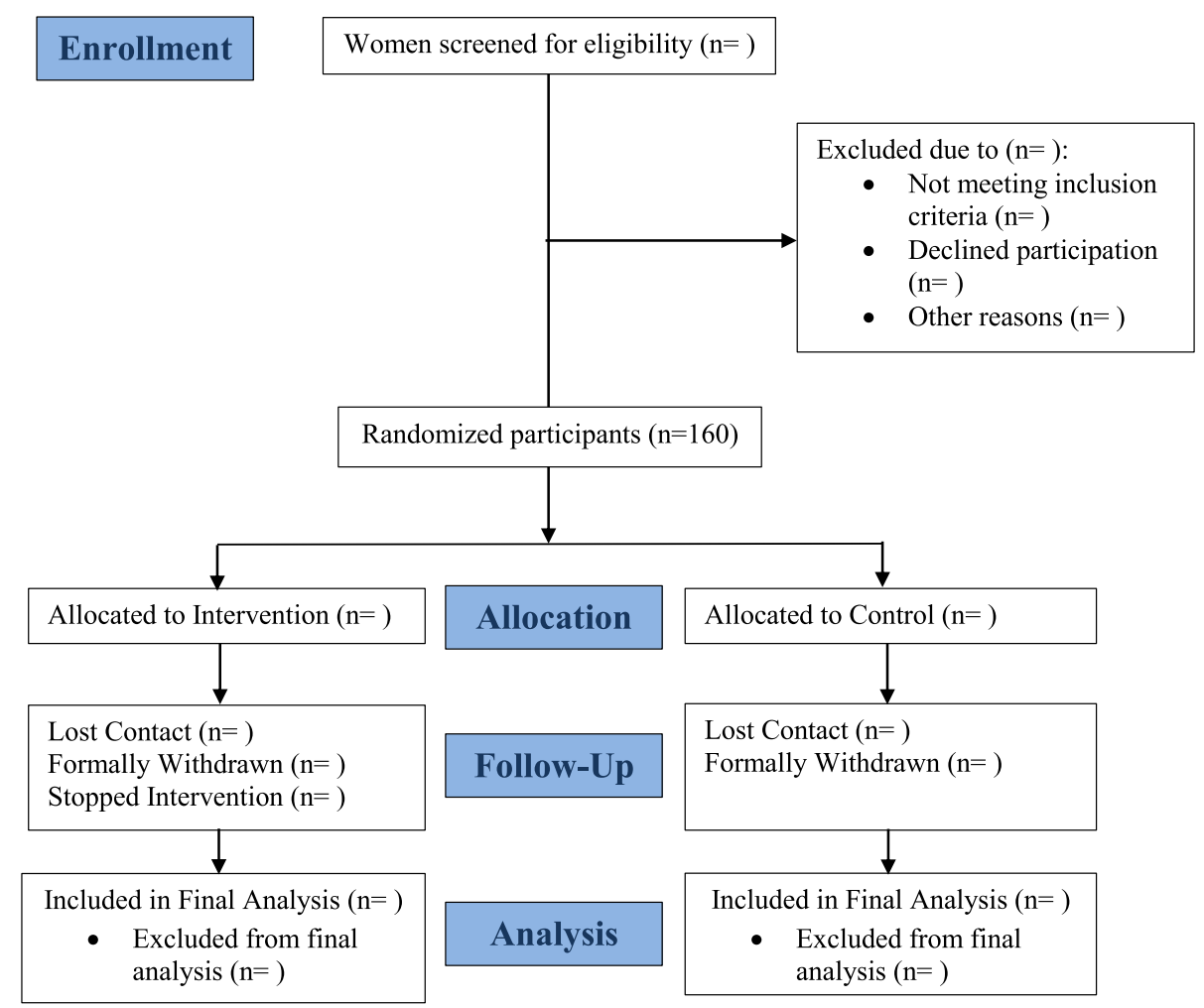

Fig. 1 CONSORT Flow Diagram (Moms in Motion Study, Columbus, Ohio, USA)

collect demographic information and data regarding current health (e.g., delivery outcomes, medical history). They will also collect initial measures of health behavior, lifestyle habits [24], motivation and confidence for behavior change [25, 26], perceptions of body shape [27], infant feeding practices, sleep quality [28], mental health and depressive symptoms [29], and perceived stress [30]. Study staff will measure the participant's weight and body composition using the Tanita TBF-310GS Body Composition Analyzer (previous model), and they will measure hip circumference and waist circumference using metric tape. Before conducting measurements, study staff will complete anthropometric measurement training to ensure consistency and accuracy.

During the visit, the participant will receive an ActiGraph wGT3X-BT accelerometer to wear for all waking hours during a 7-day run-in period after Visit 1 . Women who wear the accelerometer for more than $49 \mathrm{~h}$ over the 7 -day run-in period will be invited to continue in the study; others will be dismissed.

\section{Visit 2}

Visit 2 will occur 25-35 days postpartum in clinical research facilities at Nationwide Children's Hospital in Columbus, Ohio (Table 2). Body measurements, including weight, will be repeated. A registered nurse will collect biomarker measurements of insulin resistance via venipuncture, including a fasting 2 -h, $75 \mathrm{~g}$ oral glucose tolerance test (OGTT); insulin; hemoglobin A1c; lipid panel; adiponectin; leptin; and high sensitivity $\mathrm{C}$-reactive protein. A point-of-care glucose test will be performed at the beginning of each visit and will have a threshold of $250 \mathrm{mg} / \mathrm{dL}$; if a participant's fasting glucose is above the threshold, the principal investigator and registered nurses will investigate the situation to determine whether the participant should be withdrawn or rescheduled. The body composition and biomarker measurements completed at this visit will serve as the baseline measures. All Visit 1 questionnaires will be completed for a second time with the addition of the Diet History Questionnaire III (DHQ III). All participants will wear the ActiGraph wGT3X-BT accelerometer for 21 days after Visit 2 to monitor activity and energy expenditure. Study staff will share all blood test results with the participant and their OB/GYN or primary care provider via a letter sent in the mail stating that the results were collected for a research study and that the research staff will not provide follow-up care. Study staff will encourage participants to follow-up with their providers to discuss their results.

\section{Randomization and blinding}

Randomization will occur at the end of Visit 2. Research staff determine a participant's eligibility before Visit 2 . A 
Table 1 Overview of Protocol Measures (Moms in Motion Study, Columbus, Ohio, USA)

\begin{tabular}{|c|c|c|c|c|c|c|c|c|}
\hline \multirow{2}{*}{$\begin{array}{l}\text { Domains of Protocol } \\
\text { Measures }\end{array}$} & \multirow[t]{2}{*}{ Methodology } & \multirow[t]{2}{*}{ Data Source } & \multicolumn{5}{|c|}{ Point of Data Collection } & \multirow[t]{2}{*}{ Data Collection Methods } \\
\hline & & & $\begin{array}{l}\text { Visit } \\
1\end{array}$ & $\begin{array}{l}\text { Visit } \\
2\end{array}$ & $\begin{array}{l}\text { Check- } \\
\text { in } \\
\text { Calls } \\
\text { (4) }\end{array}$ & $\begin{array}{l}\text { Visit } \\
3\end{array}$ & $\begin{array}{l}\text { Follow- } \\
\text { Up Call }\end{array}$ & \\
\hline \multirow[t]{4}{*}{$\begin{array}{l}\text { Demographics and } \\
\text { Medical Health } \\
\text { Measures }\end{array}$} & $\begin{array}{l}\text { Contact and sociodemographic factors (age, ethnicity, } \\
\text { race, birthplace (country), average monthly income, } \\
\text { government food assistance, insurance, parity, } \\
\text { relationship status) }\end{array}$ & \multirow[t]{4}{*}{ Surveys } & $x$ & & & & & REDCap software \\
\hline & Medical History, Current Health, and Health Behavior & & $x$ & $x$ & $X(4)$ & $x$ & $x$ & REDCap software \\
\hline & Delivery Outcomes & & $x$ & & & & & REDCap software \\
\hline & New pregnancy & & $x$ & $x$ & $X(4)$ & $x$ & $x$ & REDCap software \\
\hline \multirow{5}{*}{$\begin{array}{l}\text { Body Composition and } \\
\text { Physical Activity } \\
\text { Measures }\end{array}$} & Weight (kg) & \multirow[t]{4}{*}{$\begin{array}{l}\text { In-person exam } \\
\text { with study staff }\end{array}$} & $x$ & $x$ & & $x$ & & $\begin{array}{l}\text { Tanita TBF-310GS Body } \\
\text { Composition Analyzer }\end{array}$ \\
\hline & Percent body fat (\%) & & $x$ & $x$ & & $x$ & & $\begin{array}{l}\text { Tanita TBF-310GS Body } \\
\text { Composition Analyzer }\end{array}$ \\
\hline & Waist-hip circumference and ratio & & $x$ & $x$ & & $x$ & & $\begin{array}{l}\text { Anthropometry performed } \\
\text { by trained study staff }\end{array}$ \\
\hline & Infant health (weight and length) & & & $x$ & & $x$ & & $\begin{array}{l}\text { Anthropometry performed } \\
\text { by trained study staff; } \\
\text { infant scale and } \\
\text { infantometer }\end{array}$ \\
\hline & Energy expenditure and physical activity & $\begin{array}{l}\text { ActiGraph } \\
\text { wGT3X-BT } \\
\text { device }\end{array}$ & $x$ & $x$ & & $x$ & & $\begin{array}{l}\text { ActiLife/Centrepoint Sync } \\
\text { software }\end{array}$ \\
\hline \multirow{14}{*}{$\begin{array}{l}\text { Glycemia and } \\
\text { Associated Biomarkers } \\
\text { of Insulin Resistance } \\
\text { Measures }\end{array}$} & Glucose (mg/dl) & \multirow{14}{*}{$\begin{array}{l}\text { In-person exam } \\
\text { with Clinical } \\
\text { Research } \\
\text { Services }\end{array}$} & & $x$ & & $x$ & & Blood test \\
\hline & Oral Glucose Tolerance Test & & & $x$ & & $x$ & & Blood test \\
\hline & Insulin (pmol/l) & & & $x$ & & $x$ & & Blood test \\
\hline & HOMA & & & $x$ & & $x$ & & Blood test \\
\hline & Hemoglobin A1c (\%) & & & $x$ & & $x$ & & Blood test \\
\hline & Total cholesterol (mg/dl) & & & $x$ & & $x$ & & Blood test \\
\hline & Triglycerides (mg/dl) & & & $x$ & & $x$ & & Blood test \\
\hline & HDL-cholesterol (mg/dl) & & & $x$ & & $x$ & & Blood test \\
\hline & LDL-cholesterol (mg/dl) & & & $x$ & & $x$ & & Blood test \\
\hline & Systolic blood pressure $(\mathrm{mmHg})$ & & & $x$ & & $x$ & & Vital sign \\
\hline & Diastolic blood pressure $(\mathrm{mmHg})$ & & & $x$ & & $x$ & & Vital sign \\
\hline & Adiponectin $(\mu \mathrm{g} / \mathrm{ml})$ & & & $x$ & & $x$ & & Blood test \\
\hline & Leptin (ng/ml) & & & $x$ & & $x$ & & Blood test \\
\hline & hsCRP (mg/dl) & & & $x$ & & $x$ & & Blood test \\
\hline \multirow{8}{*}{$\begin{array}{l}\text { Psychosocial Outcome } \\
\text { Measures }\end{array}$} & Health Promoting Lifestyle Profile II & \multirow[t]{8}{*}{ Surveys } & $x$ & $x$ & $X(1)$ & $x$ & $x$ & REDCap software \\
\hline & Motivation/Confidence for Behavior Change & & $x$ & $x$ & $X(1)$ & $x$ & $x$ & REDCap software \\
\hline & Body Shape Questionnaire & & $x$ & $x$ & $X(1)$ & $x$ & & REDCap software \\
\hline & Diet History Questionnaire III & & & $x$ & & $x$ & & Web-based survey tool \\
\hline & Breast/Infant Feeding & & $x$ & $x$ & $X(4)$ & $x$ & $x$ & REDCap software \\
\hline & Pittsburgh Sleep Quality Index & & $x$ & $x$ & $X(1)$ & $x$ & $x$ & REDCap software \\
\hline & Depression (CES-D) & & $x$ & $x$ & $X(1)$ & $x$ & & REDCap software \\
\hline & Perceived Stress (PSS-10) & & $x$ & $x$ & $X(1)$ & $x$ & & REDCap software \\
\hline $\begin{array}{l}\text { Process Outcome } \\
\text { Measures }\end{array}$ & $\begin{array}{l}\text { Intervention acceptability, study satisfaction, suggestions } \\
\text { for improvements }\end{array}$ & Surveys & & & $X(1)$ & $x$ & $x$ & REDCap software \\
\hline
\end{tabular}

Table 1 outlines the protocol measures, methodology, data sources, points of data collection, and data collection methods that will be utilized in this study

pseudorandom number generator in the statistical software package SAS will be used to implement randomization, stratified by study clinic and BMI group with randomly varying block sizes. MAK will maintain the randomization sequence that will be unavailable to other research study members. Women will be randomized 1:1 to either the intervention or the control condition, stratified by clinic site and BMI category (18.5-24.9 
Table 2 Visit Schedule (Moms in Motion Study, Columbus, Ohio, USA)

\begin{tabular}{llll}
\hline Study Visit & Days Postpartum & Days Post-Randomization & Acceptable Window \\
\hline Visit 1: Home Visit & $7-14$ days & Not applicable & $7-14$ days postpartum \\
Visit 2: First Clinic Visit & 30 days & 0 days & $25-35$ days postpartum \\
Visit 3: Final Clinic Visit & 220 days & 190 days & $190-210$ days post-randomization \\
\hline
\end{tabular}

or $\geq 25$ ). The stratifying variables will be entered into REDCap by the research staff, and the concealed randomization algorithm will be programmed in REDCap and used by research staff to randomize women sequentially. Blinding of study investigators, staff, and women will not be possible with this intervention.

There will be five data collection calls. Table 3 shows the timeline for which these calls will be completed and the acceptable windows for these calls to be completed. Table 1 details the measures included in each data collection call.

\section{Phone call data collection}

Women will participate in a series of data collection calls and surveys via a REDCap email link at 90, 120, 150, and 180 days postpartum (Table 3 ). During the phone calls, study staff will answer all study-related questions and document any health changes that may have occurred since their last encounter. Staff will encourage women to continue participating (i.e., continue wearing ankle weights, completing ankle weight journal logs, and completing surveys) by reinforcing the value of their participation.

\section{Visit 3}

Visit 3 will occur 190-210 days post-randomization in clinical research facilities at Nationwide Children's Hospital (Table 2). It will repeat all measures from Visit 2. The body composition and biomarker measurements at this visit will serve as the final measures. As with Visit 2, study staff will share all blood test results with the participant and their OB/GYN or primary care provider via a letter sent in the mail stating that the results were collected for a research study and that the research staff will not provide follow-up care. Study staff will encourage participants to follow-up with their providers to discuss their results.

\section{Final phone contact (follow-up phone call)}

Women will participate in a final data collection call and surveys via a REDCap email link 360 days postpartum (Table 3). During the phone call, the participant will complete questionnaires, and study staff will collect data on their satisfaction with the study, intervention acceptability, and suggestions for improvements.

\section{Description of the intervention}

Women assigned to the intervention will receive the standard recommendation to engage in $150 \mathrm{~min}$ of physical activity per week from their obstetric care provider. They will also receive a pair of ankle weights ( 2.5 pounds [1.1 kg] per ankle) after randomization during Visit 2 to wear until Visit 3. Study staff will instruct women to wear the ankle weights for $2 \mathrm{~h}$ every day during routine daily activities (i.e., childcare, household chores) and document the date, time, and activities completed in a study journal for the duration of the intervention. Women will be reminded and encouraged to send pictures of their study journal via email or text message. Study staff will send reminders as encouragement and appreciation weekly when they send in their journals.

Women assigned to the control arm will receive the standard recommendation from their obstetric care provider to engage in $150 \mathrm{~min}$ of physical activity per week. They will not receive a pair of ankle weights to wear after randomization, and they will not complete a study journal. This was selected as the control condition because it is the current standard of care.

\section{Primary outcome Weight loss}

The Tanita TBF-310GS Body Composition Analyzer will measure weight. Women will be weighed wearing light clothing and no shoes nor socks. We will account for 1 $\mathrm{kg}$ of clothing weight for every participant during each measurement. Postpartum weight loss will be defined as weight change between Visits 2 and 3.

\section{Secondary outcomes \\ Body composition}

Height will be abstracted from the EMR before Visit 1 from the vitals section, which may be a measured or self-reported height. The Tanita will measure percent body fat, and trained study staff will measure waist and hip circumference using a metric tape. Waist circumference $(\mathrm{cm})$ will be assessed at the middle point between the ribs and the iliac crest, with the participant in a standing position. Hip circumference $(\mathrm{cm})$ will be measured at the widest circumference of the buttocks. Change in body composition measurements such as body fat percentage, BMI, and waist-hip ratio will be defined as the measurement change between Visits 2 and 3. The infant's length and weight will be measured at 
Table 3 Data Collection Call Schedule (Moms in Motion Study, Columbus, Ohio, USA)

\begin{tabular}{lll}
\hline Data Collection Call & Days Postpartum & Days Post-Randomization \\
\hline Check-in Call \#1: End of Month 2 & 90 days & $60-65$ days \\
Check-in Call \#2: End of Month 3 & 120 days & $90-95$ days \\
Check-in Call \#3: End of Month 4 & 150 days & $120-125$ days \\
Check-in Call \#4: End of Month 5 & 180 days & $150-155$ days \\
Follow-Up Phone Call & 360 days & $330-340$ days \\
\hline
\end{tabular}

Visits 2 and 3 using a calibrated infantometer and an infant scale.

\section{Glycemia and associated biomarkers}

The clinical laboratory at Nationwide Children's Hospital will analyze all blood samples collected, except for adiponectin and leptin which will be sent to a partner laboratory. Insulin sensitivity and $\beta$-cell function will be measured using the Matsuda index94 and HOMA-IR.

\section{Potential mediators}

\section{Energy expenditure and physical activity}

All women will receive an ActiGraph wGT3X-BT accelerometer to monitor their daily activity and energy expenditure. Using a belt clip, women will wear the ActiGraph on their waist for 21 days after Visit 2 and for 21 days before Visit 3. All devices will be calibrated based on the participant's anthropometric data and configured for recording. Study staff will encourage women to sync their device daily to a phone application via Bluetooth technology. The application will upload data to a software system called CentrePoint, and only study staff will see the participant's personal online profile in that system to allow for regular monitoring of compliance. For participants without access to a smartphone or Bluetooth technology, study staff will manually upload their data to hospital computers upon arrival at Visits 2 and 3 . Women will receive customized reminder communications asking them to wear their ActiGraph upon request or if study staff observe that the participant is not wearing the device as often as desired. All participants will be blinded, such that no physical activity data can be viewed.

\section{Diet}

Participants will complete the Diet History Questionnaire III (DHQ III), a web-based food frequency questionnaire based on a collection of national 24-h dietary recall data from the National Health and Nutrition Examination Surveys (NHANES) conducted from 2007 to 2014 .

\section{Psychosocial assessments}

Participants will complete surveys to assess changes in healthy lifestyle habits [24], sleep quality [28], motivation for behavior change $[25,26]$, perceptions of body shape [27], infant feeding practices, mental health and depressive symptoms [31], and perceived stress [30].

\section{Analysis}

The study will adhere to CONSORT statement guidelines using intent-to-treat as the primary approach for analysis. All individuals will be kept in the group to which they were randomized, regardless of protocol violations or dropouts. Only the pre-specified covariates of baseline outcome variable, race, pre-pregnancy BMI category, GDM severity, parity, clinic location, and education will be included as covariates in the primary and secondary analyses unless imbalances are detected across arms in baseline variables [32]. Balance between the treatment groups will be tested by informally comparing baseline variables between study arms. Outliers will be checked and kept in the final analysis. Outcome variables will also be examined over time at the group and the individual level graphically as well as via descriptive statistics stratified on time and group. No interim analyses or stopping guidelines are planned regarding data monitoring.

Furthermore, bivariate relationships between outcome variables and potential covariates will be examined to determine if substantial deviations from linearity exist. We will make an effort to minimize dropouts and missing data, but if substantial missing data is realized, maximum likelihood estimation will be employed. Although not expected, if substantial non-compliance is realized, modern methods for handling missing data will be utilized [33, 34]. We will continue to collect all outcome data from participants who discontinue the intervention as long as the participant does not formally withdraw and is not lost to follow-up.

Analyses for the primary and secondary outcomes will involve a mixed effects model for repeated measures, similar to ANCOVA but based on maximum likelihood [35]. The same approach, statistical models, and time points (i.e., Visit 2 and 3) used for our primary hypothesis will be used for our secondary hypotheses, with each body composition and biomarker outcome in separate models instead of weight loss. For the Exploratory Aim, we will employ a longitudinal mediation model with multiple mediators. We will use Mplus 7.31 with 
bootstrapping procedures to statistically test mediators. We will follow the guidelines for testing longitudinal mediation within Cole and Maxwell and MacKinnon $[36,37]$. These tests are important for clinical applicability of the intervention by identifying the most salient mechanisms of change that ultimately lead to better outcomes in our target population. Multiple publications will be submitted to peer-reviewed journals to disseminate study results.

\section{Participant safety and monitoring and data management} Human subjects review and approval are under the $\mathrm{Na}$ tionwide Children's Hospital's Institutional Review Board (IRB) with a reliance agreement from the Ohio State University Medical Center's IRB. The IRB has deemed the study "minimal risk." Experienced research nursing staff will perform biospecimen collections and monitor patients during oral glucose tolerance tests. Study staff will assess adverse events at each study visit and during each data collection call via online surveys using REDCap software. The principal investigator and study doctor will review the adverse events monthly unless suspected to be Serious Adverse Events, and they will determine what action will be taken due to the adverse event regarding the study intervention. The study investigators will inform the IRB of all adverse events and will report all serious and related adverse events on an expedited timeframe per IRB guidelines.

The study will follow all necessary measures to assure the participant data stored in the database are protected and secure from unauthorized access. Only team members who are approved by the IRB to have access to the data will have access. Data use agreements with external collaborators may be possible as long as they are within the scope of the informed consent form. There is no planned auditing from the funder or other outside entity. Internal audits may be conducted on an unannounced basis.

\section{Discussion}

The Moms in Motion study is innovative in its emphasis on simplicity to accelerate health improvement during a time when attention to self-health is not easily attainable. All new mothers experience physical and lifestyle changes and unavoidable time constraints postpartum, making it challenging to initiate sustainable weight loss efforts, which are essential for women with GDM to prevent/delay a T2DM diagnosis. The occurrence of GDM and high postpartum weight retention [1] continually contribute to the increasing number of women with T2DM. This study was designed to test an intervention to reduce T2DM risk among women who had GDM by considering the postpartum demands new mothers experience.
By testing an intervention that is sensitive to the postpartum demands new mothers experience, requiring minimal time and effort, we can shift current clinical paradigms to facilitate early weight loss and reduce T2DM risk among women with GDM. Beyond the expectation of this study to be significant in its direct health impacts from weight loss, it will be significant in exploring (1) the mechanism(s) by which the intervention is successful (mediating effects of energy expenditure and/or diet on weight loss) and (2) the effects of the intervention on body composition and biomarkers associated with insulin resistance and metabolic health.

Although this study is ongoing, we will expect the findings to be meaningful regarding the effectiveness of the intervention and how to engage women with GDM in the postpartum period to reduce T2DM risk. A limitation of this study is that we lack the resources to translate and validate questionnaires and other materials into other languages, so we must exclude women who do not speak English, which may reduce the generalizability of the findings. Also, participants will be followed to 12 months' postpartum, but current resources do not permit longer-term follow-up. Future directions could incorporate following the participants over an extended period, beyond the duration of this study, to observe continued or sustained weight loss. Future research could also involve conducting a similar intervention in other settings or with larger samples adequately powered to robustly examine mechanisms.

\section{Abbreviations}

GDM: Gestational diabetes mellitus; T2DM: Type 2 diabetes mellitus; OGTT: Oral glucose tolerance test; DPP: Diabetes Prevention Program; RCT: Randomized controlled trial; PI: Principal investigator; IRB: Institutional Review Board

\section{Acknowledgements}

We would like to thank our participants and their families for volunteering their valuable time to our study. We would like to acknowledge Lisa Buccilla, RN, Brenda Widmayer, RN, and Julie Somppi, RN for their help with study recruitment. We would also like to thank the Clinical Research Services staff at Nationwide Children's Hospital for their help with administering and managing the clinic visits.

\footnotetext{
Authors' contributions

SAK and ROF wrote and designed the study protocol, and they serve as Principal Investigators. SAK serves as the trial sponsor. SFT and MBL contributed to study design and conception of the study protocol. SMB and BJS drafted, edited, and completed initial drafts of the manuscript. BJS completed the final draft of the manuscript under the supervision of SAK and ROF. MAK designed the randomization sequence used in the procedures. KKG assisted with editing initial and final drafts of the manuscript. All authors assisted substantially with critically revising initial drafts of the manuscript. All authors substantively revised, read and approved the final submitted version of this manuscript and all previous versions that involved the author's contribution to the study. All authors have agreed to be personally accountable for their own contributions and have ensured that questions related to the accuracy or integrity of any part of the work are appropriately investigated, resolved, and the resolution documented in the literature.
} 


\section{Funding}

This work is supported by the American Diabetes Association (grant \#1-18JDF-062). The study protocol, design, and approach were externally peer reviewed as part of the American Diabetes Association funding award process. This source was not involved in the design of the study and collection, analysis, and interpretation of data nor in writing the manuscript.

\section{Availability of data and materials}

Not applicable.

\section{Declarations}

\section{Ethics approval and consent to participate}

The study was approved by Nationwide Children's Hospital's Institutional Review Board (IRB \#18-00178), and the study has a reliance agreement with the Ohio State University Wexner Medical Center. Informed consent takes place in private at 30-40 weeks' gestation or 7-14 days postpartum during Visit 1. Consent obtained from study participants was initially written consent but was approved by the IRB to also be verbal when in-person visits were limited (starting March 2020 due to the COVID-19 pandemic).

\section{Consent for publication}

Not applicable.

\section{Competing interests}

The authors declare that they have no competing interests.

\section{Author details}

${ }^{1}$ Center for Biobehavioral Health, The Research Institute at Nationwide Children's Hospital, 700 Children's Drive, Columbus, OH 43205, USA. 2Department of Pediatrics, College of Medicine, The Ohio State University, 370 W. 9th Avenue, Columbus, OH 43210, USA. ${ }^{3}$ Division of Epidemiology, College of Public Health, The Ohio State University, 250 Cunz Hall, 1841 Neil Avenue, Columbus, OH 43210, USA. ${ }^{4}$ Department of Obstetrics and Gynecology, College of Medicine, The Ohio State University, 370 W. 9th Avenue, Columbus, $\mathrm{OH} 43210$, USA. ${ }^{5}$ Center for Perinatal Research, The Research Institute at Nationwide Children's Hospital, 700 Children's Drive, Columbus, OH 43205, USA. 'Department of Pediatric Endocrinology, Nationwide Children's Hospital, 700 Children's Drive, Columbus, OH 43205, USA. ${ }^{7}$ Ohio Department of Health, 246 N High Street, Columbus, OH 43215, USA.

Received: 11 March 2021 Accepted: 20 May 2021

Published online: 29 June 2021

\section{References}

1. Casagrande SS, Linder B, Cowie CC. Prevalence of gestational diabetes and subsequent type 2 diabetes among US women. Diabetes Res Clin Pract. 2018;141:200-8. https://doi.org/10.1016/j.diabres.2018.05.010.

2. Bellamy L, Casas J-P, DHingorani A, Williams D. Type 2 diabetes mellitus after gestational diabetes: a systematic review and meta-analysis. Lancet. 2009:373(9677):1773-9. https://doi.org/10.1016/S0140-6736(09)60731-5.

3. Holmes VA, Draffin CR, Patterson CC, Francis L, Irwin J, McConnell M, et al. Postnatal lifestyle intervention for overweight women with previous gestational diabetes: a randomized controlled trial. J Clin Endocrinol Metabol. 2018;103(7):2478-87. https://doi.org/10.1210/jc.2017-02654.

4. Ackermann RT. Diabetes prevention at the tipping point: aligning clinical and public health recommendations. Ann Intern Med. 2015;163(6):475-6. https://doi.org/10.7326/M15-1563.

5. Bernstein J, Quinn E, Ameli O, Craig M, Heeren T, Iverson R, et al. Onset of T2DM after gestational diabetes: what the prevention paradox tells us about risk. Prev Med. 2018;113:1-6. https://doi.org/10.1016/j.ypmed.2018.05.005.

6. Di Cianni G, Lacaria E, Lencioni C, Resi V. Preventing type 2 diabetes and cardiovascular disease in women with gestational diabetes-the evidence and potential strategies. Diabetes Res Clin Pract. 2018;145:184-92. https:// doi.org/10.1016/j.diabres.2018.04.021.

7. James E, Freund M, Booth A, Duncan MJ, Johnson N, Short CE, et al. Comparative efficacy of simultaneous versus sequential multiple health behavior change interventions among adults: a systematic review of randomised trials. Prev Med. 2016;89:211-23. https://doi.org/10.1016/j. ypmed.2016.06.012.
8. Verplanken B, Melkevik O. Predicting habit: the case of physical exercise. Psychol Sport Exerc. 2008;9(1):15-26. https://doi.org/10.1016/j.psychsport.2 007.01.002.

9. Ferrara A, Hedderson MM, Albright CL, Ehrlich SF, Quesenberry CP, Peng T, et al. A pregnancy and postpartum lifestyle intervention in women with gestational diabetes mellitus reduces diabetes risk factors: a feasibility randomized control trial. Diabetes Care. 2011;34(7):1519-25. https://doi. org/10.2337/dc10-2221.

10. Ferrara A, Hedderson MM, Brown SD, Albright CL, Ehrlich SF, Tsai A-L, et al. The comparative effectiveness of diabetes prevention strategies to reduce postpartum weight retention in women with gestational diabetes mellitus: the gestational diabetes' effects on moms (GEM) cluster randomized controlled trial. Diabetes Care. 2016;39(1):65-74. https://doi.org/10.2337/dc1 5-1254.

11. Nicklas JM, Zera CA, England LJ, Rosner BA, Horton E, Levkoff SE, et al. A web-based lifestyle intervention for women with recent gestational diabetes mellitus: a randomized controlled trial. Obstet Gynecol. 2014;124(3): 563-70. https://doi.org/10.1097/AOG.0000000000000420.

12. Saligheh M, McNamara B, Rooney R. Perceived barriers and enablers of physical activity in postpartum women: a qualitative approach. BMC Pregnancy Childbirth. 2016;16(1):131. https://doi.org/10.1186/s12884-0160908-X.

13. Tang JW, Foster KE, Pumarino J, Ackermann RT, Peaceman AM, Cameron KA. Perspectives on prevention of type 2 diabetes after gestational diabetes: a qualitative study of Hispanic, African-american and white women. Matern Child Health J. 2015;19(7):1526-34. https:// doi.org/10.1007/s10995-014-1657-y.

14. Downs DS, Hausenblas HA. Women's exercise beliefs and behaviors during their pregnancy and postpartum. J Midwifery Women's Health. 2004;49(2): 138-44. https://doi.org/10.1016/j.jmwh.2003.11.009.

15. Cheung NW, Blumenthal C, Smith BJ, Hogan R, Thiagalingam A, Redfern J, et al. A pilot randomised controlled trial of a text messaging intervention with customisation using linked data from wireless wearable activity monitors to improve risk factors following gestational diabetes. Nutrients. 2019;11(3):590. https://doi.org/10.3390/nu11030590.

16. Peacock A, Bogossian FE, Wilkinson S, Gibbons K, Kim C, Mclntyre H. A randomised controlled trial to delay or prevent type 2 diabetes after gestational diabetes: walking for exercise and nutrition to prevent diabetes for you. Int J Endocrinol. 2015;2015:1-8. https://doi.org/10.1155/2015/423717.

17. Calfas KJ, Marcus BH. Postpartum weight retention: a mother's weight to bear? Am J Prev Med. 2007;32(4):356-7. https://doi.org/10.1016/j.amepre.2 007.01.008.

18. Healy GN, Dunstan DW, Salmon J, Cerin E, Shaw JE, Zimmet PZ, et al. Objectively measured light-intensity physical activity is independently associated with 2-h plasma glucose. Diabetes Care. 2007;30(6):1384-9. https://doi.org/10.2337/dc07-0114.

19. Borodulin K, Evenson KR, Herring AH. Physical activity patterns during pregnancy through postpartum. BMC Womens Health. 2009;9(1):32. https:// doi.org/10.1186/1472-6874-9-32.

20. Ainsworth BE, Haskell WL, Herrmann SD, Meckes N, Bassett DR Jr, TudorLocke C, et al. 2011 compendium of physical activities: a second update of codes and MET values. Med Sci Sports Exerc. 2011;43(8):1575-81. https://doi. org/10.1249/MSS.0b013e31821ece12.

21. Evenson KR, Herring AH, Wen F. Self-reported and objectively measured physical activity among a cohort of postpartum women: the PIN postpartum study. J Phys Act Health. 2012;9(1):5-20. https://doi.org/10.1123/ jpah.9.1.5.

22. Bassuk SS, Manson JE. Epidemiological evidence for the role of physical activity in reducing risk of type 2 diabetes and cardiovascular disease. J Appl Physiol. 2005;99(3):1193-204. https://doi.org/10.1152/japplphysiol.001 60.2005.

23. Carpenter MW, Coustan DR. Criteria for screening tests for gestational diabetes. Am J Obstet Gynecol. 1982;144(7):768-73. https://doi.org/10.1016/ 0002-9378(82)90349-0.

24. Walker $\mathrm{S}$. Health promotion model - instruments to measure health promoting lifestyle : health promoting lifestyle profile [HPLP II] (adult version). Omaha: College of Nursing, Community-Based Health Department, University of Nebraska Medical Center; 1995. https://deepblue.lib.umich.edu/handle/202 7.42/85349.

25. Stetson B, Schlundt D, Peyrot M, Ciechanowski P, Austin MM, Young-Hyman $D$, et al. Monitoring in diabetes self-management: issues and 
recommendations for improvement. Popul Health Manage. 2011;14(4):18997. https://doi.org/10.1089/pop.2010.0030.

26. Skouteris H, McCabe M, Milgrom J, Kent B, Bruce LJ, Mihalopoulos C, et al. Protocol for a randomized controlled trial of a specialized health coaching intervention to prevent excessive gestational weight gain and postpartum weight retention in women: the HIPP study. BMC Public Health. 2012;12(1): 78. https://doi.org/10.1186/1471-2458-12-78.

27. Stunkard A, Sørensen T, Schulsinger F. Use of the Danish adoption register for the study of obesity and thinness. Res Publ-Assoc Res Nerv Ment Dis. 1983;60:115

28. Buysse DJ, Reynolds CF III, Monk TH, Berman SR, Kupfer DJ. The Pittsburgh sleep quality index: a new instrument for psychiatric practice and research. Psychiatry Res. 1989;28(2):193-213. https://doi.org/10.1016/0165-1781 (89)90047-4.

29. Radloff LS. The CES-D scale: a self-report depression scale for research in the general population. Appl Psychol Meas. 1977;1(3):385-401. https://doi.org/1 $0.1177 / 014662167700100306$

30. Cohen S, Kamarck T, Mermelstein R. A global measure of perceived stress. J Health Soc Behav. 1983;24(4):385-96. https://doi.org/10.2307/2136404.

31. Radloff LS. The CES-D scale: a self-report depression scale for research in the general population. Appl Psychol Meas. 1977;1(3):17.

32. Senn S. Covariate imbalance and random allocation in clinical trials. Stat Med. 1989;8(4):467-75. https://doi.org/10.1002/sim.4780080410.

33. Schafer JG, Graham JW. Missing data: our view of the state of the art. Psychol Methods. 2002;7(2):147-77. https://doi.org/10.1037/1082-989X.7.2.14 7.

34. Little R, Yau L. Intent-to-treat analysis for longitudinal studies with dropouts. Biometrics. 1996;52(4):1324-33. https://doi.org/10.2307/2532847.

35. Winkens B, van Breukelen GJ, Schouten HJ, Berger MP. Randomized clinical trials with a pre- and a post-treatment measurement: repeated measures versus ANCOVA models. Contemp Clin Trials. 2007;28(6):713-9. https://doi. org/10.1016/j.cct.2007.04.002.

36. Cole DA, Maxwell SE. Testing mediational models with longitudinal data: questions and tips in the use of structural equation modeling. J Abnorm Psychol. 2003:112(4):558-77. https://doi.org/10.1037/0021-843X.112.4.558

37. Mackinnon DP. Introduction to statistical mediation analysis. New York: Taylor \& Francis; 2008.

\section{Publisher's Note}

Springer Nature remains neutral with regard to jurisdictional claims in published maps and institutional affiliations.

Ready to submit your research? Choose BMC and benefit from:

- fast, convenient online submission

- thorough peer review by experienced researchers in your field

- rapid publication on acceptance

- support for research data, including large and complex data types

- gold Open Access which fosters wider collaboration and increased citations

- maximum visibility for your research: over $100 \mathrm{M}$ website views per year

At $\mathrm{BMC}$, research is always in progress.

Learn more biomedcentral.com/submissions 